\title{
La Enseñanza Virtual del Derecho. Una aproximación en la Universidad cubana
}

The virtual teaching of the right. An approximation at the university Cuban.

\author{
Alcides Antúnez Sánchez \\ Licenciado en Derecho. \\ Máster en Asesoría Jurídica. \\ Profesor Auxiliar Derecho Ambiental e Internacional. \\ Departamento de Derecho. \\ Facultad de Ciencias Económicas y Sociales. \\ Universidad de Granma. República de Cuba. \\ Contacto: aantunez@udg.co.cu; antunez63@nauta.cu
}

\section{Armando Antúnez Sánchez}

Médico Veterinario.

Profesor Auxiliar Tecnologías Educativas.

Máster en Nuevas Tecnologías para la Educación.

Experto Nacional en Educación a Distancia.

Universidad de Granma. República de Cuba.

Contacto: antunez@udg.co.cu

\section{Yolanda Soler Pellicer}

Licenciada en Cibernética-Matemática.

Doctora en Ciencias Técnicas.

Profesora Titular Ciencias Informáticas.

Facultad de Ciencias Naturales e Informática.

Universidad de Granma. República de Cuba.

Contacto: yoly@udg.co.cu 


\section{Resumen}

El artículo realiza un esbozo de los referentes teóricos que se han tenido en cuenta para el desarrollo de las habilidades en el proceso docente educativo, las principales ideas desde un enfoque histórico-cultural, se fundamenta la necesidad e importancia de la formación de los estudiantes de Derecho para crear en habilidades sociales que le permitan demostrar sus competencias en el mercado laboral. Es realizada para ello una revisión de la introducción de la actividad científica dentro del proceso de enseñanza-aprendizaje que le permita el desarrollo de habilidades en la Universidad Cubana. A partir de la perspectiva de las nuevas tecnologías digitales en el desarrollo cognitivo de los jóvenes en sus valores, estilos de vida y expectativas educativas, para apropiarse de conocimientos o saberes jurídicos, y dotarlos de una serie de competencias y habilidades básicas que lo capaciten para el ejercicio de su profesión una vez que este acceda al mercado laboral desde los modos de actuación como fiscal, juez, abogado, asesor, profesor, entre otros. Para ellos nos auxiliamos en los métodos de la investigación como el histórico lógico, el de análisis y síntesis, y el de inducción deducción.

\section{Palabras clave}

Competencias laborales, habilidades, proceso docente educativo.

\section{Abstract}

The article sells off a sketch of the referent theoreticians that have been had in account for the development of the abilities in the teaching educational process, the principal ideas from a historic cultural focus, the need and importance of the formation to create in these social abilities that be enabled to demonstrate his competitions on the market labor are based. He is once a revision of the introduction of the scientific activity within the process of teaching was accomplished for it learning that the development of abilities at the University Cuban enable him. As from the perspective of the new digital technologies in young people's cognitive development in his moral values, styles of life and educational expectations, in order to take possession of knowledge or juridical knowledges, and to endow them of a series of competitions and basic abilities that they qualify it to the exercise of his profession once this come over the labor market from the modes of acting like district attorney, judge, lawyer, adviser, professor, between other ones. We helped ourselves in the methods of the investigation for them like: The historic logician, the one belonging to analysis and synthesis, and the one belonging to induction deduction.

\section{Keywords}

Labor competitions, abilities, teaching educational process. 


\section{Notas introductorias}

La tarea de formar e informar a los juristas de estos tiempos debe ser una preocupación de todos, se trata de formar un profesional que esté vinculado a la problemática social, para que pueda comprender la naturaleza real del Derecho como ciencia, valorado como un fenómeno en sí mismo, donde se logre combinar propósitos prácticos y profesionales con otros estrictamente teóricos o científicos, como es el caso de la aplicación de las herramientas que nos aporta la informática.

El sistema de conocimientos, en la enseñanza del Derecho, no tiene sentido sin la existencia de los mecanismos de su estructuración, conservación y aplicación; por eso en el desarrollo del pensamiento aparecen las habilidades como expresión de la sistematización de procedimientos, técnicas y métodos. Son estructuras del pensamiento, lógicas, motoras, que permiten asimilar, conservar, utilizar y exponer los conocimientos. Se estructuran en operaciones que constituyen su estructura técnica. Se forman y desarrollan a través de la ejercitación de las acciones y se convierten en modos de actuación que dan solución a tareas teóricas y prácticas. Álvarez (1997).

También el modo de interacción del sujeto con los objetos, se dan en la actividad y la comunicación, constituyen el contenido de las acciones que el sujeto realiza con un objetivo, juegan un papel importante en la autorregulación del sujeto y en el conocimiento de sí mismo. Fuentes (1999).

El impacto tecnológico-científico, llamado como la 3ra Revolución industrial, la industrialización, el desafío demográfico, el cambio social dirigido o espontáneo, se valora que son los elementos catalizadores que impactan a la Universidad tradicional con el desarrollo de los Tics, las que inciden en los Entornos Virtuales Académicos (EVA). Todo esto motiva a los autores del ensayo, para delimitar las habilidades que deben formarse en estudiantes de la carrera de Derecho, partiendo de su análisis teórico como categoría del proceso docente en relación con las Tics.

En el artículo se realiza una caracterización de cómo se articula la enseñanza del Derecho en la Universidad cubana en el proceso docente educativo en la formación de habilidades en los estudiantes, en el uso Tics en los EVA, lo que coadyuvará una menor presencialidad y contribuirá al acceso de un mayor número de ciudadanos en cumplimiento de un principio del Derecho Constitucional del acceso gratuito a la educación.

No está sustentado en ningún proceso de investigación que, de salida a acciones de posgrado o tesis, ni en algún proyecto que lo financie, su fin es acercarnos a los EVA en la formación del profesional del Derecho, como meta futura en la educación superior cubana en el proceso de actualización del modelo económico social. Fueron utilizados los métodos de investigación como el histórico, el de análisis síntesis, el exegético jurídico e inducción deducción.

\section{1. ¿Cómo definir las habilidades?}

Para hablar de la naturaleza y clasificación de los contenidos del proceso de enseñanza aprendizaje, definen a la habilidad como: "Uno de los tipos de contenido, junto con los conocimientos y las aptitudes". De Zayas (1997).

Otros la informan como el "Sistema de acciones y operaciones dominadas por el hombre, estructuradas en operaciones ordenadas y orientadas a la consecución de un objetivo, que le permiten interactuar con objetos determinados de la realidad y con otros 
sujetos. La habilidad es el modo de interacción del sujeto con el objeto, es el contenido de las acciones que el sujeto realiza, integradas por un conjunto de operaciones, que tiene un objetivo y que se asimila en el proceso." Fuentes (1998).

Las habilidades, se adquieren en un proceso de apropiación de la estructura del objeto sobre el que recae la acción, de modo que en semejante proceso se desarrollan habilidades en el acto de aprender conocimientos y se aprenden conocimientos en el acto de desarrollar habilidades. Rondón Valdez, Antúnez Sánchez (2016).

En esta definición queda delimitado que en la habilidad están presentes el sujeto que interacciona desarrollando la habilidad, el objeto o sujeto sobre el que interactúa el primero, el objetivo con que actúa y un sistema de operaciones. Otros plantean como componente de la habilidad a su imagen generalizada o base orientadora de la acción, considerando que la habilidad se debe asimilar a partir de una imagen de las acciones a realizar dada de antemano. Talízina (1984).

Lo cual es contraproducente, ya que, si la habilidad debe ser erigida en el propio proceso de enseñanza aprendizaje, generalizando el estudiante la misma en su actividad e interacción con el grupo, la imagen generalizada se construye conjuntamente con la habilidad durante el proceso y no como parte de la estructura de la habilidad en sí. Se puede concretar que la estructura de la habilidad consta de: sujeto (el que realiza la acción), objeto (el que recibe la acción del sujeto) y el objetivo (aspiración consciente del sujeto) - sistema de operaciones (estructura técnica de la habilidad).

Analizando los criterios señalados por Talízina (1984) al caracterizar la habilidad atendiendo a sus elementos: además del conjunto de operaciones que la integran y constituyen su estructura técnica, hay que tener en cuenta al sujeto que debe dominar la habilidad, el objetivo que se satisface mediante la habilidad, la orientación que determina la estructura de dicha acción y el resultado que se ha de esperar de la acción (que tiene que coincidir con el objetivo).

Lo que permitirá analizar up supra la clasificación de las habilidades dentro de la estructura como sistema del proceso enseñanza-aprendizaje en la formación académica.

\section{Clasificación de las habilidades en el proceso enseñanza-aprendizaje}

Dentro de la estructura del sistema de habilidades, se precisa una clasificación del sistema de habilidades, a partir de tipificaciones dadas por Talízina (1984) y Álvarez (2001); según Coll (2008), las habilidades se deben clasificar en tres grupos a saber: habilidades específicas, propias de las ciencias, de las profesiones o de las tecnologías que son objeto de estudio o trabajo, habilidades lógicas o intelectuales, habilidades de comunicación.

Existen otras clasificaciones, pero en todas se aprecia como hay como rasgo común: el que las habilidades son el resultado de un proceso de aprendizaje, que tienen que transitar por determinados niveles de la sistematización. Sí consideramos un proceso de formación inductivo y no deductivo obtendremos como resultado el desarrollo de las capacidades de los estudiantes. El primer nivel de habilidad, en una ciencia o tecnología dada, lo identificamos como la habilidad elemental; ésta se sustenta en conocimientos de esa ciencia, tecnología o arte y en habilidades primarias, que actúan como operaciones dentro de esta habilidad elemental. 
Sólo las habilidades lógicas, están siempre constituidas por operaciones que también son lógicas. Esto se fundamenta en trabajos realizados por investigadores, en que se justifica el carácter inseparable de las habilidades lógicas en el proceso del pensamiento, donde se dan de manera integrada, existiendo una mutua dependencia entre ellas. Hay una interrelación entre el conocimiento y la habilidad; en particular, en las habilidades lógicas se constata como requieren consideraciones especiales.

El vínculo entre habilidad y conocimiento, no queda en la habilidad primaria y la habilidad elemental; en la medida en que se van sistematizando las habilidades también se sistematizan los conocimientos. En el propio proceso se dan ambas, sobre la base de la sistematización de las habilidades se logrará la de los conocimientos.

Las habilidades lógicas (del pensamiento), no se corresponden de manera directa, con una determinada disciplina o ciencia concreta, sino que cada disciplina puede y debe contribuir a su formación. Claro está, cada disciplina formará aquellas habilidades lógicas que se avienen a las características del objeto de estudio de la misma.

Las habilidades lógicas son múltiples, sólo las más usadas constituirían un gran listado, pero es posible a partir de la formación intencional de algunas de ellas, asegurar la formación del pensamiento lógico, pues estas habilidades no se dan de forma aislada, sino muy relacionadas entre sí durante el proceso de su apropiación y aplicación a los contenidos de las disciplinas.

En el proceso de apropiación del contenido, están presentes el análisis, la síntesis, la comparación, la abstracción, etc. entrelazadas mutuamente. Para algunos "el pensamiento se dirige para resolver los problemas a los procesos mentales vinculados entre sí y que se funden uno en otro." Rubinstein (2002).

La relación conocimiento-habilidad específica y de pensamiento lógico, se va perfeccionando en dos direcciones en el proceso de enseñanza-aprendizaje, construyendo habilidades de mayor nivel de sistematicidad. Estas se clasifican en:

I. A través de un proceso consciente que permite cumplir acciones teóricas y prácticas de mayor complejidad, lo cual se produce en el enfrentamiento de mayor riqueza.

II. A partir de un proceso de ejercitación, donde enfrentan problemas de igual grado de complejidad, de manera que se automaticen las habilidades, siendo el sujeto cada vez menos consciente de sus acciones, formando un hábito.

Permitirá abordar la formación de algunas habilidades dentro de la carrera de Derecho en el modelo de enseñanza virtual, a partir del plano teórico y práctico como meta futura del modelo educativo cubano en la educación superior.

\section{La enseñanza virtual del Derecho. Una aproximación desde la Universidad cubana}

Luego de hacer un abordaje preliminar de las habilidades, se precisa su vinculación con la enseñanza virtual del Derecho en la nación cubana, desarrollada en este ensayo jurídico.

Se constata que los estudios históricos del Derecho en Cuba son tan antiguos como la misma enseñanza universitaria, al fundarse la Real y Pontificia Universidad de San Jerónimo, entre sus primeras carreras figuró la de la licenciatura en Derecho, con un fuerte ingrediente de subsistencia del antes llamado Trivium y Cuatrivium. Durante la etapa colonial, la historia jurídica señala que estos estudios estuvieron marcados por la influencia absoluta de la perspectiva filosófica y académica del escolasticismo, contra 
el cual tuvo que quebrar lanzas desde la cátedra de Derecho Constitucional del Seminario de San Carlos y San Ambrosio en la actual capital de la nación Varela (1996, pp. 34-182), Torres Cuevas (2002), Fernández Viciedo (2014).

En los años de la República neocolonial, en los estudios de Derecho se aprecia como oscilaron entre su adscripción a un pensamiento iusnaturalista casi pietista, absolutamente idealista y políticamente liberal burgués o un pensamiento positivista, el que era francamente normativista desde la década de los años cuarenta del siglo XX, en el que fue casi literalmente indetenible la influencia de la obra de Kelsen (2009).

Al aprobarse en 1976, la Constitución Socialista y fundarse el Ministerio de Educación Superior, se procedió al diseño del primer Plan de estudios con ambiciones pedagógicas y científicas, llamado Plan A por el Ministerio de Educación Superior. A la luz de la Constitución y de resultas del trabajo, primero de las Comisiones de Estudios Jurídicos, y después del esfuerzo de la Asamblea Nacional del Poder Popular, se aprobó un buen número de normativas jurídicas que fueron completando el sistema de derecho cubano y brindaron apoyatura absoluta a los esfuerzos académicos y científicos en esta rama de las ciencias sociales en el ordenamiento jurídico.

De ese modo, con este antecedente se diseñó el Plan B, y en el cual se vencían limitaciones, errores e insuficiencias del anterior Plan A. Este Plan se mantuvo en vigor, con varios cambios y adecuaciones, hasta la década de los años 80 del pasado siglo.

Le sustituyó en este tracto el Plan de Estudio C, constituyó el resumen de la voluntad académica encaminada a brindar un alto contenido científico a los estudios de Derecho, desligándolos de los lastres normativistas y positivistas que pesaron sobre la formación y el ejercicio profesional durante varias décadas en Cuba, concebido bajo el principio de "enseñar Derecho y no solo legislación". Fernández Bulté (1997).

El Plan de Estudio de Derecho C, se renueva con el D en el 2008, este se desarrolló con la misma línea tendencial que sus antecesores, pero a criterio de los autores en la materia de informática y el Derecho, se constata que se mantiene la tendencia de los planes de estudios que le han antecedido en este particular, al no estar concebida en el el Derecho Informático.

En este ir y venir, ante la situación a la que se enfrenta la población mundial a principios del milenio, el desafío para los educadores, al ser los encargados de formar las nuevas generaciones para cumplir su encargo social en este siglo XXI, donde en esta misma cuerda, con la masificación de la tecnología, el desenvolvimiento vertiginoso de la ciencia, la innovación y el cambio en la información científica, como fenómenos que se dan a una velocidad imposible de registrar, le corresponde a la educación transformarse en "aprender a aprender", como la única manera de seguir con relativa fluidez el mundo dinámico y complejo de la actualidad, como la estrategia del aprendizaje que permita evaluar la competencia. Delors (1994), Bates (2001, pp.25-45), Martin Ortega (2008, pp.72-78), Jornet (2011), Cobas Cobiella (2016).

Se pondera, que se requerirá de un alto grado de habilidades como: la observación, la obtención de información, la planificación de la misma, la solución de problemas y la comunicación de sus resultados, entre otras que, como procedimientos generales, permitirán profundizar en el conocimiento de la realidad y determinar sus características, establecer sus nexos, sus regularidades, es decir, poder conocer y fundamentar lo válido o no por los estudiantes en el proceso enseñanza-aprendizaje.

Estos elementos, exigen una Universidad y un profesor que aplique en el proceso de enseñanza-aprendizaje en la formación del estudiante de Derecho, los métodos y pro- 
cedimientos de la investigación, la dinámica de la ciencia y, sobre todo, estos exigen mayor sistematización e integración de los resultados de la investigación, los que se apropian con las habilidades en el quehacer del estudiante, para que su resultado final sea un profesional con competencias en su desempeño, donde aparecen en este escenario las habilidades que se crean con la implementación y uso de las Tics en los EVA. Antúnez Sánchez, Soler Pellicer (2015).

En el debate sobre el impacto de la Revolución tecnológica en el mundo en el desarrollo en general, y en América Latina en particular, el tema del rol del Estado no puede estar ausente; no sólo porque el Estado sigue siendo para bien o para mal un actor fundamental de la vida política, económica y social de los pueblos, sino porque al igual que todas las organizaciones de la sociedad actual éste está siendo profundamente transformado por el cambio tecnológico como un paradigma social. Se acota que "la difusión de la tecnología amplifica infinitamente su poder al apropiársela y redefinirla sus usuarios. Las Tics no son sólo herramientas que aplicar, sino procesos que desarrollar. Castells (1999, p.48)

Se pondera que, desde los finales del siglo pasado, Sebastia (1996) identificaba a las tecnologías de la información como "...un nuevo perfil y una nueva nomenclatura..." y a su vez propuso, para designar a la formación de los profesionales y de los usuarios de la información, el término: infotecnologías, pero no es hasta el año 2001, cuando se consolida este desarrollo tecnológico, integrándose con todas las esferas de la sociedad.

Este año se fija como el inicio de la nueva Internet, conocida como la Web 2.0, la que tiene como premisa tecnológica el soporte de la Red Universal Digital (RUD), aunque algunos autores prefieran la denominación de Nuevo Entorno Tecno social (NET) el cual conforma la nueva versión de Internet: “Este Nuevo Entorno supondría una singular novedad en la breve pero acelerada historia de la Era de la Información, a la que se ha llegado, al menos parcialmente, gracias a un proceso de evolución sociedadtecnología o infotecnologías". Cabero Almanera (2007), Soler Pellicer (2015).

Dentro del desarrollo de las Tics, están las infotecnologías, las que ya no son sólo un nuevo perfil o disciplina, sino que también son parte de la "cultura tecnológica", entendida por la cultura, como un conjunto de "conocimientos y habilidades prácticas" de los individuos, los cuales son indispensables para mantener relaciones exitosas con el nuevo entorno y con otros individuos. Fumero, Roca, Sáez-Vacas (2007), Cabero Almanera (2009).

Esta postura se corrobora como contrasta con las visiones conclusivas resultantes de la Cumbre Mundial de la "sociedad de la información" (2003), y la Declaración de Principios de Ginebra, adoptada por los gobiernos, la cual expresa en su primer artículo: "Nosotros... declaramos nuestro deseo y compromiso comunes de construir una "sociedad de la información" centrada en la persona, integradora y orientada al desarrollo, en la que todos puedan crear, consultar, utilizar y compartir la información y conocimiento para que las personas, las comunidades y los pueblos puedan emplear plenamente sus posibilidades en la promoción de su desarrollo sostenible y en la mejora de su calidad de vida, sobre la base de los propósitos y principios de la Carta de las Naciones Unidas, en respeto pleno y en defensa de la Declaración Universal de Derechos Humanos (1949), donde se reconoce los derechos de 4ta generación". Sanz Larruga (1996). 
Es un hecho de que las Tics aún no son objeto de estudio por parte de las distintas disciplinas jurídicas, es el caso como ya se ha citado, del Derecho Informático dentro del Derecho como ciencia social, en la enseñanza de pregrado. Empero, los rápidos avances de estas tecnologías producen notables transformaciones en la sociedad de la información, que han de ser objeto de regulación por parte del Derecho como ciencia en cuerpos jurídicos dentro del ordenamiento jurídico, para poder establecer un marco legal adecuado que dé respuesta a los problemas jurídicos que se plantean como consecuencia de las mismas, ponderándose con ello el principio de legalidad desde el Derecho Público. Delpiazzo (2009).

Entrar en ese "mundo social y tecnológico" requiere la formación de la población en general con ciertas habilidades en el uso de las Tics, que parten de educar colectivamente a los beneficiarios de estas tecnologías como factores de crecimiento, igualdad e inclusión social, donde entra a jugar un rol esencial los EVA a criterio de los autores, al aplicarse el modelo de la triple hélice, pero son necesarios los conocimientos básicos para dominar estas tecnologías y las habilidades adquiridas para poder asimilarlas e implementarlas. Sánchez Rodríguez (2005), Antúnez Sánchez (2016).

Al respecto, las Tics pueden jugar un papel fundamental en el proceso de conceptualización y ejecución de políticas públicas del Estado; su impacto puede producir avances significativos en diversos sectores, no sólo desde el punto de vista de la participación social en los niveles formales de la sociedad, sino como promotor del desarrollo social, la inclusión ciudadana, la transparencia en la gestión pública en los servicios públicos y el fortalecimiento de la participación ciudadana, todo discursa a través de la plataformas infotecnológicas, las que demandan partidas presupuestarias para su concreción social en la materia educativa. Delpiazzo (2009).

El siglo XXI, muestra nuevos retos para la enseñanza superior en las naciones, uno de estos en la transformación del proceso de enseñanza aprendizaje en la carrera de Derecho. Autores como Duart y Sangrá (2000), han señalado que el uso de las Tics en los espacios universitarios, permite el desarrollo de tres elementos a quienes lo utilizan, con mayor flexibilidad e interactividad; la vinculación con los docentes y el resto del alumnado permiten mayor colaboración y participación y la facilidad para acceder a los materiales de estudio y a otras fuentes complementarias de información, y es aquí donde aparece el EVA.

Otros autores como Cabero Almanera y Llorente (2005) reseñaron que el uso de las tecnologías en los centros educativos favorece en los educandos los medios para la adquisición de las destrezas tecnológicas, las que se requieren en la actual sociedad de la información y del conocimiento, también han notificado que se amplía el acceso al aprendizaje, el que se mejorará con la calidad de la enseñanza y se aprecia el desarrollo y expansión de algunas tecnologías en este sector.

Delgado y Solano (2009) consideraron que los EVA, permiten una flexibilización de las estructuras docentes, propiciando nuevas concepciones en el aprendizaje en la que los cursistas, tienen un papel más activo y protagónico y al mismo tiempo trabajan de forma colaborativa; por otro lado los docentes asumen el papel de facilitadores y deben encontrar nuevas estrategias que permitan mantener activos a los participantes cuando estos se encuentren en distintos sitios, promoviendo la construcción de conocimientos y la colaboración.

Se aprecia como Flores (2012), describió que las tecnologías van favoreciendo una serie de cambios y transformaciones en las formas en que se desarrollan los procesos 
formativos. Precisamente, una de estas herramientas son los EVA, los que constituyen un escenario óptimo para promover espacios que integran diversas herramientas a través de los ordenadores conectados a la red, permitiendo la realización de los procesos de enseñanza y aprendizaje en un ambiente pedagógico y metodológico específico. Autores como Soler, Antúnez, Mercado y Ramírez (2015) significaron que en la actualidad, las Universidades deben promover experiencias innovadoras en las actividades de postgrados apoyadas en el uso de las tecnologías contrarias al modelo tradicional que por muchos siglos ha sido implementado en las aulas de manera presencial, lo que ha permitido demostrar el énfasis que debe hacerse en la docencia, en los cambios de estrategias didácticas de los profesores, en los sistemas de comunicación y distribución de los materiales de aprendizaje, en lugar de enfatizar la disponibilidad y las potencialidades de las tecnologías. Se ha notificado que los docentes son los actores que mayores cambios pueden generar en los centros educativos. Por otra parte, en las Universidades se están desarrollando programas virtuales en acciones de pregrado y postgrado.

Análisis que, permitirán adentrarnos en la aplicación de las infotecnologías y su incidencia en la formación del estudiante, como un activo que a través de la relación en el proceso docente educativo a los futuros profesionales que se vincularan a las formas de gestión públicas y privadas, para su posterior desarrollo empresarial en los EVA, para poder acceder a barreras comerciales exigentes en el mundo corporativo del siglo XXI, como uno de los paradigmas a resolverse por la Administración Pública para alcanzar el desarrollo sostenible en este mundo de la híper conectividad global. Lee (2008).

De esta forma, se evidencia un enfoque social de la ciencia y la tecnología (CTS) por parte del Estado, pues se logra articular estrategias de instrucción mediante la implementación de mecanismos de alfabetización científica y tecnológica de los ciudadanos, a fin de alcanzar la participación y promover la amplia e irrestricta inclusión digital, informacional y social en todas las capas de la sociedad. Ferreira y Didzack, citado por Rincón y Romero (2007).

En consecuencia, todos los análisis realizados en torno a los criterios acerca de la definición de que es la Infotecnología y para que se implementa, se justiprecia como es a principios del siglo XXI, donde se afirmara que: "El buen manejo de los computadores y de la Internet es una de las habilidades que deben caracterizar al ciudadano competente en el siglo XXI. Lograr entonces que al terminar su etapa escolar los jóvenes dominen las herramientas básicas de las Tics es un objetivo importante del plan curricular de cualquier institución educativa". Piedrahita Plata (2003).

Es por ello, que se preconiza que una adecuada preparación con esta herramienta al capital humano a partir de la formación académica, dependerá de resultados exitosos a las formas de gestión que las apliquen al tener la habilidad desarrollada, vinculadas en su aplicación y utilidad a las ciencias contables, en la protección ambiental, al desarrollo productivo, a la implementación de tecnologías limpias, en el desarrollo del comercio interno y foráneo, aportando valores añadidos en el mundo de la competencia empresarial "marketing empresarial". Ya reseñada con la aplicación por los sujetos de gestión en la implementación de la Responsabilidad Social Empresarial -4ta Revolución Industrial- Antúnez Sánchez (2016), Liliana Coria (2017).

La enseñanza virtual del Derecho, dentro del sistema pedagógico moderno, recurre a la tecnología informática para fortalecer el aprendizaje, la investigación, el autoestu- 
dio, la interacción y la flexibilidad de los estudios, permitiendo a los profesionales obtener una o varias profesiones, posgrados, especialidades, superando las barreras del tiempo y espacio para desarrollarse profesionalmente y contribuir al desarrollo de la sociedad, en su desarrollo se aplican las habilidades y conocimientos adquiridos a través del uso de las Laptop, Tablet y Móviles androide. Stable-Gutiérrez (2006, pp.18-26) Sus antecedentes en el sistema de la educación nacional patrio, se considera ubicarlo en el curso por correspondencia antes del modelo social actual; y a partir del triunfo de la Revolución con el modelo semipresencial. Donde el estudiante de manera individual se prepara para ser evaluado, se utiliza para ello libros en forma digital, lo que le permite gestionar los conocimientos con el uso de las Tics.

Es valorado con ello, que el ambiente telemático se configura a partir de la irrupción en nuestra sociedad de las Tics. La barrera de espacio y tiempo propia del ser humano se rompe, originando una nueva forma de enfrentarse y entender la propia existencia y la interacción con los otros y con lo otro. Donde, la llamada "sociedad de la información y el conocimiento", ante la generación de un volumen insospechado de información en el mundo empresarial, constituye un reto para los profesionales, la que debe utilizarse con responsabilidad, siendo esta parte del control público ejecutado por la Administración Pública.

Al resultar imprescindible la formación continua de estos profesionales para salvar la brecha de la infoxicación, y discernir entre el enorme volumen de información existente y la de alta calidad; se pondera como también constituye una prioridad desarrollar en el profesional capacidades que le permitan guiarse a través del intrincado laberinto de información y obtener aquella que precisa para desplegar con rigor su actividad profesional dentro de las formas de gestión donde tenga relación jurídica laboral. Machado Ramírez (2014).

Es una realidad que, en los escenarios internacionales y nacionales, el uso de las Tics hoy constituye un elemento indispensable en el desarrollo de todos los aspectos de la vida, estos se aprecian desde la búsqueda de información hasta la comunicación personal y la aplicación de las herramientas de la Web 2.0. Cabero Almanera (2008).

Los autores consideran, que es también un medio donde la educación no se encuentra ajena al uso e implementación de las Tics, donde cada vez se aprecia que se descubre un universo ilimitado de posibilidades, brindando toda una gama de herramientas para el aprendizaje con la posibilidad de expandirse a un número de usuarios cada vez mayores, en diferentes escenarios y con la capacidad de socializar este conocimiento adquirido.

Se aprecia que esta tecnología digital, está permitiendo la creación de entornos más flexibles para el aprendizaje y favorecen el trabajo colaborativo entre los educandos, rompiendo con los escenarios formativos tradicionales, ofreciendo nuevas posibilidades para las orientaciones y la tutoría de los alumnos y facilitar una formación permanente. Todos estos nuevos espacios telemáticos, permiten crear entornos de comunicación sincrónicos y asincrónicos, facilitando eliminar las barreras espacio-temporales entre las personas, potenciando contextos interactivos. Salinas (2002), Carabantes (2005), Cabero Almanera (2008).

Es un hecho, que en las diversas carreras que se estudian en la Educación Superior cubana, donde el modelo del profesional se precia privilegiado con la habilidad de gestionar información y conocimientos, al potenciársele esta habilidad del uso de las Tics, lo cual es básico y necesario para poner al estudiante en contacto con el objeto de la pro- 
fesión desde los primeros años de la carrera en la que este estudie, entre las que se pueden señalar el ingeniero en Ciencias informáticas, el ingeniero industrial, el jurista, el médico, el estomatólogo, entre otras carreras.

Lo que permitirá sistematizar con estas habilidades, la implementación de la estrategia curricular para el manejo adecuado de la información científico-técnica, mediante el uso de las Tics, como una cuestión clave para asegurar un desempeño laboral exitoso, donde se desempeñen como futuros profesionales en el rol que se ubiquen en las formas de gestión en la actualización del modelo económico y social (personas jurídicas o naturales), como ha sido reseñado en el desarrollo de ensayo. Reyes Pérez (2017), Pineda Pacheco (2017).

Desde esta perspectiva, se pondera que el dominio adecuado del proceso de gestión de la información, como parte esencial de la dimensión desarrolladora en el proceso formativo del profesional. Estos profesionales para resolver los problemas de la profesión, requieren de aplicar la investigación científica, la que no solo se limita a los profesionales de los centros de investigación, sino que se aplica en otros contextos. Es aquí, donde aparece el egresado con habilidades en el uso de las Tics, quien al realizar en su función tareas investigativas relacionadas con la búsqueda de alternativas y el perfeccionamiento, contribuirá también a transformar la sociedad en que convive como ser social. Rico Carrillo (2007), Salinas Arata (2012).

Uno de los ámbitos que no ha escapado a la influencia de las Tics, es el de la enseñanza superior y en especial la de la carrera de Derecho. En efecto, la aplicación de estas tecnologías en todos los niveles de la enseñanza supone nuevos retos y oportunidades en los métodos y procesos de aprendizaje, teniendo la calidad como objetivo. En consecuencia, también en el ámbito universitario se ha visto la necesidad de incorporar las Tics para poderlas adaptar a las transformaciones sociales que se acometen en la universidad cubana. De ahí que las Tics hayan abierto la posibilidad de utilizar formas innovadoras de enseñar y aprender el Derecho como ciencia. García (2007).

Es criterio de los autores, que es este uno de los paradigmas que hoy tiene como reto la nueva Universidad en el siglo XXI, graduar estudiantes con competencias para el mercado laboral, del que no están exentos los profesionales del Derecho con el uso de las Tics. Lo que trae como colofón, reseñar que el desarrollo de la capacitación al capital humano dentro de las formas de gestión en el escenario mercantil cubano, como parte de la formación académica en las Universidades es un elemento positivo a explotarse, que permita el desarrollo a futuro de las formas de gestión, y con ello dar mejores respuestas a los empresarios y asumir retos en el comercio ante mercados cada vez más exigentes en este siglo XXI, para el cumplimiento de las normas administrativas para el uso del Derecho Informático en el derecho sustantivo, donde hoy se pondera la aplicación de las Tics y la certificación empresarial con la norma ISO 26000 . Antúnez Sánchez (2016).

Se pondera, que una empresa que en este siglo XXI, que no tenga en cuenta que la globalización de la actividad económica, la que impone nuevos desafíos sin precedentes a la competitividad empresarial, que ha dejado de basarse en ventajas estáticas, con frecuencia relacionadas con la dotación de recursos naturales, el clima, o el mecanismo de formación de precios y el ajustes de costos (ambientales, tributarios, salariales, etc.), para vincularse directamente a la dinámica de la incorporación de infraestructura tecnológica y del conocimiento novedosas, traducido en nuevos procesos o productos, con nuevas formas de gestión y nuevas competencias laborales; donde con 
el uso de las Tics le facilitan su construcción, difusión y la articulación de procesos decisorios y productivos, y con ello la generación de valores para la innovación en los procesos de investigación, producción, y comercialización de sus riquezas en segundos, como es aplicado en la Empresa de Alta Tecnología en la nación cubana. Antúnez Sánchez (2016).

Es el caso del uso del e-Learning, valorada como la plataforma que permite de manera rápida y eficaz adquirir habilidades y conocimientos profesionales, la que, combinada a los últimos avances de la tecnología multimedia, permite activar los sentidos de los usuarios que la utilizan en las formas de gestión para el desarrollo del capital humano, está considerada como el gran activo de la empresa, donde el EVA es ponderado como herramienta eficaz y eficiente. Stable-Gutiérrez (2006, pp.18-26), Cabero Almanera (2007).

Se constata, como en el Plan de Estudios de la carrera de Derecho en el sistema nacional de educación, no se establece una asignatura en su currículo que estudie en el pregrado el Derecho Informático, solo está concebido un Taller de computación con 32 horas lectivas -Introducción a la Computación-. Este taller se dirige a formar en el estudiante las habilidades básicas para el uso gradual y progresivo de las ventajas de las novedosas técnicas de la información y la comunicación.

Se forma en ellos las habilidades básicas para el uso gradual y progresivo de las ventajas de las novedosas técnicas de la información y la comunicación; de forma tal que desde el primer año de la carrera el estudiante pueda procesar textos; crear presentaciones con incorporación de diferentes objetos; enviar y recibir mensajes textuales o no, con uso de ficheros adjuntos, de firmas, etc.; navegar y buscar información entre diferentes sitio en Internet; manejar la mensajería electrónica como medio de comunicación; construir páginas y sitios web de poca complejidad; y crear aplicaciones para la gestión de información.

Para afianzar estas habilidades señaladas, se aplica la estrategia curricular homónima en la que la intervienen las restantes disciplinas orientando y exigiendo la aplicación de dichas técnicas en relación con la búsqueda de información científico técnica actualizada; la presentación de exposiciones y de trabajos de investigación curriculares o extracurriculares.

Ahora bien, en la carpeta de las asignaturas electivas, tampoco se aprecia como aparece establecida como una materia a impartir en la carrera el Derecho Informático, donde a criterio de los autores puedan ser abordados temáticas como: la cibernética, la informática jurídica, la sociedad informatizada, seguridad informática, informática y derechos patrimoniales, informática jurídica documental, de gestión, decisional, informática y derechos humanos, los bienes informáticos, la contratación y los servicios informáticos, la prueba informática, la contratación electrónica, los negocios jurídicos automatizados, y los tipos de responsabilidad en materia informática, entre otros; empero se reafirma según diseño curricular que se garantizará que los conocimientos adquiridos en la computación se conviertan desde el primer momento en una herramienta habitual de trabajo de los estudiantes; exigiéndose resultados y aplicaciones durante toda la carrera en la elaboración de tareas extraclases, trabajos de curso, procesamiento de resultados obtenidos en prácticas laborales, búsqueda de información bibliográfica, confección de documentos, gráficos y presentaciones en PowerPoint. Para ello también deberá asegurarse la utilización de la intranet; la Internet, las páginas web y de laboratorios virtuales creados para apoyar las diferentes formas de enseñan- 
za y el desarrollo de competencias profesionales. Deben también desde un inicio formar hábitos con relación a la seguridad informática, lo que sin la información señalada no pueden salir graduados con todas las competencias. Pérez Luño (1998, p.722), Antúnez Sánchez (2009), Delpiazzo (2009), Palomar Olmeda (2009).

Esto conlleva también, a que los profesores con las categorías académicas y científicas -másteres y doctores en Derecho-, aparte de manejar el software común de la carrera de Derecho, deberán estar dispuestos a adquirir conocimientos de la plataforma digital a utilizar por la institución académica en el uso de las infotecnologías, la que es perceptible de llegar a variar el destino dado a las herramientas de dicha plataforma.

En esta plataforma digital, deberán aparecer todas y cada una de las materias del Plan de estudios, las que deben ser muy explícitas, ya que el alumnado hacia el cual va dirigida esta enseñanza virtual del Derecho, buscará claridad en cuanto a la forma de trabajar, esto le permitirá sentirse en un ambiente de confianza y en consecuencia aprovechar mejor los recursos disponibles, dando mejores resultados.

De ahí la importancia que, a criterio de los autores de contar con las políticas públicas del curso a desarrollarse, la metodología a utilizar, las formas de evaluación, y para la bibliografía, sobre todo, se deberá contar con una estructura en los sistemas que sea ágil, con la capacidad suficiente para no tener interferencias para la adecuada búsqueda de información en los EVA, para romper paradigmas en este siglo XXI. Cirilo Aguilar (2011, pp. 74-79).

Se precia que, cada módulo debe tener actividades a realizar que permitan reforzar con práctica lo aprendido y al final una actividad integradora en la que se relacionen todos y cada uno de los temas impartidos. Por lo que la discusión de la enseñanza del Derecho en forma tradicional de la clase magistral o la implementación del estudio o análisis de casos, estaría sujeta a su implementación para romper los paradigmas en la enseñanza virtual del Derecho en los nuevos EVA, válidos para la formación del pregrado y para la actividad del posgrado en la universidad cubana del siglo XXI.

Se preconiza, que las Tics han hecho posible la aparición en las intranets universitarias (Moodle) de espacios de interrelación entre el profesor y los alumnos, esto es, las webs de las asignaturas, que constituyen, sin duda, una herramienta pedagógica de apoyo o complemento a la docencia universitaria presencial y para la semipresencial. En estos espacios los estudiantes pueden encontrar información, materiales y recursos didácticos relevantes para el desarrollo de la asignatura (como el programa, criterios de evaluación, noticias de interés, bibliografía básica...), enviar sus ejercicios prácticos, consultar sus calificaciones, participar en debates, etc. Incluso, el profesor puede utilizar la web de la asignatura para canalizar las tutorías o consultas de la asignatura (dudas relativas al funcionamiento de la asignatura o a su contenido). Bauzá Reilly (1995, pp.31-44).

Es sin dudas, el instrumento tecnológico que puede servir para lograr incentivar el interés del estudiante por una materia jurídica y facilitar su aprendizaje, aminorando la carga de trabajo para el estudiante, a la vez que le familiarizará con los EVA en la implementación de las habilidades. Es una ayuda importante para que los estudiantes organicen de forma autónoma su estudio y avancen adecuadamente en los contenidos de la asignatura indicados por su profesor.

Es también importante superar las tradiciones y costumbres medievales, y tratar de orientar la enseñanza utilizando la participación activa de los estudiantes, usando los medios tecnológicos y culturales más ágiles, para que los profesionistas se preparen de 
manera de desenvolverse adecuadamente, ya que con resistencias o sin ellas enfrentarán de inmediato una competencia despiadada que exige su mejor preparación y la ampliación de sus conocimientos necesarios para su formación en pre y posgrado.

Por ello, el paso más allá en la evolución de la presencialidad a la virtualidad viene constituido por las asignaturas que presentan horas de docencia virtual, en las que a partir de los materiales que el profesor pone a disposición de los alumnos, éstos pueden estudiar los contenidos de la asignatura. Nieto Göller (2012, pp.137-150).

En este mismo contexto, también es utilizado el Blended learning o enseñanza mixta, tiene la ventaja como sistema, que de forma inmediata el tutor o profesor pueden hacer los comentarios pertinentes a través de un chat previamente agendado por ambos para aclarar las dudas y hacer las sugerencias necesarias, esto independiente de la tutoría académica permanente en el horario ya establecido. Cabero Almanera (2009), Antúnez Sánchez (2016).

Este espacio virtual que se configura, al que se le llama aula sin paredes, cuyo mejor exponente actual es la red de internet, no es presencial, sino representacional, no es proximal, sino distal, no es sincrónico sino multicrónico y no se basa en recintos espaciales con interior, frontera y exterior, se depende de las redes electrónicas cuyos nodos de interacción pueden estar diseminados por diversos países. Nieto Göller (2012, pp.137-150).

También dentro del curso se considera, que el estudiante y el profesor pueden enlazarse con especialistas del Derecho para el desarrollo de las videoconferencias o entrevistas, dando al alumno la certeza de estar al día en la información jurídica, se aprecia que un exponente de ello fue en Cuba el proceso de universalización de la enseñanza con el uso de la televisión y videos en el proceso docente-educativo. Quien tendrá la obligación de contactar a estos especialistas será el tutor o el profesor quien dirige el proceso docente educativo, ya que sólo él sabrá cómo va el avance del curso, su aprovechamiento, qué es necesario reforzar y, en consecuencia, si es el momento oportuno para hacerlo con responsabilidad. Morales García (2002), Valero Torrijos (2004), Romeo Casabona (2006).

Cobas Cobiella (2014) refirió que, los profesores de Derecho, casi sin ninguna excepción y profesionales en general, han sido formados bajo una enseñanza memorística, y de aprendizaje de artículos y de conceptos abigarrados, en muchas ocasiones, sin interconexión conceptual y con el trabajo meramente individual, premiando los logros individuales. Por supuesto, este tipo de enseñanza también tiene ventajas y por ello, la idea es trazar un modelo de estudio, que permita vincular lo mejor de cada sistema.

Esta autora consultada señaló que la Declaración de Bolonia (1999) Italia, firmada por los Ministros de Educación de 29 países europeos, trata la reformulación de la Educación Superior sobre la base de unos objetivos de aprendizaje comunes a cada uno de los niveles educativos (grado y postgrado). Desarrollando determinadas competencias que son necesarias y que demanda el sector empresarial, entre las que destacan la capacidad de análisis y síntesis, la capacidad de aplicar los conocimientos en la práctica; la planificación y gestión del tiempo; los conocimientos generales básicos sobre el área de estudio; los conocimientos básicos de la profesión, comunicación oral y escrita en la lengua materna, el conocimiento de una segunda lengua, habilidades básicas de manejo del ordenador, habilidades de investigación, capacidad de aprender, habilidades de gestión de la información, capacidad crítica y autocrítica, capacidad para adaptarse a nuevas situaciones, capacidad para generar nuevas ideas (creatividad), resolu- 
ción de problemas, toma de decisiones, trabajo en equipo, habilidades interpersonales, liderazgo, capacidad de trabajar en un equipo interdisciplinar, capacidad para comunicarse con personas no expertas en la materia, apreciación de la diversidad y multiculturalidad, habilidad para trabajar en un contexto internacional, conocimiento de culturas y costumbres de otros países, habilidad para trabajar de forma autónoma, diseño y gestión de proyectos, iniciativa y espíritu emprendedor, compromiso ético, preocupación por la calidad, motivación por el logro.

Por ello, se razona que las asignaturas que se desarrollan en los EVA, como en un campus virtual, en estos casos, se trata de enseñanza universitaria a distancia, asistida por medios telemáticos o redes digitales. Están requeridos de especialistas en elaboración del material didáctico, se distinguen en tres rasgos, los que definen a este tipo de docente: experto en contenidos propios de la materia que se imparte, conocedor del lenguaje hipermedia y multimedia y sobre todo conocedor de las metodologías de educación a distancia y en la repercusión que este hecho tiene para la elaboración de este tipo de materiales. Que sean autoinstructivos, autoevaluativos, y adaptados a las situaciones de aprendizaje y al perfil del aprendiz. García, L. Ruiz, M. Domínguez, D. (2007, p.20).

Se pondera que las plataformas EVA para la gestión del conocimiento y el aprendizaje, habrán de depurar sus prestaciones y ofrecer la máxima facilidad a docentes y estudiantes. A los primeros, para que con suma facilidad puedan aprovechar todas las posibilidades de la herramienta y a los segundos para que la navegación sea lo más intuitiva posible.

Otro ámbito en el que la proliferación de las Tics ha supuesto un cambio importante en el mundo jurídico y, en concreto, en el de la enseñanza universitaria del Derecho es el del tratamiento y la gestión de información con relevancia jurídica, tan necesaria en este ámbito. En este sentido, se ha pasado, por una parte, de libros y revistas doctrinales en formato papel a revistas y libros electrónicos, y, por otra, de recopilaciones en papel de jurisprudencia y normativa a bases de datos jurídicas, contenidas originariamente en CD-rom y, posteriormente, accesibles desde Internet (en línea), es decir, desde cualquier punto geográfico de un país. Cabero Almanera (2010), Antúnez Sánchez (2016).

Ejemplos de lo señalado en la nación cubana son: la Revista CubaLex de la Facultad de Derecho de la Universidad de La Habana; la Revista Cubana de Derecho del Gremio jurídico; el sitio web de la Gaceta Oficial de la República del Ministerio de Justicia, la página digital diaria del gremio jurídico difundiendo el acontecer jurídico; las Revistas de la Fiscalía General de la República y la del Tribunal Popular de la República de Cuba, el uso de las Tics en los procesos judiciales y los servicios que se prestan en los Registros del Estado Civil del Ministerio de Justicia, el sitio web de la Asamblea Nacional del Poder Popular, entre otras publicaciones y sitios digitales donde se difunde la producción científica y las disposiciones jurídicas del país.

En concreto, el impacto y uso de las Tics en la actividad de la docencia en el Derecho, se valora que provocará una serie de modificaciones en los métodos de enseñanza y de aprendizaje universitario, entre las que destacan los cambios producidos tanto en la labor del profesor universitario como en la del estudiante; con todo, no son los únicos que se dan en este ámbito en los EVA, ya que adquieren una gran importancia los materiales docentes y tiene lugar un nuevo planteamiento de la evaluación periódica y la 
sistemática, que incidirá en el aprendizaje cooperativo. Pérez Luño (2004), Cobas Cobiella (2014).

Por ello los autores de este ensayo consideran, que deberá ser objeto de estudio por los profesores de la carrera de Derecho en conjunto con la carrera de Informática, para dotar de una plataforma digital que pondere a futuro la carrera de Derecho de manera virtual, como otra opción más en la formación del ciudadano cubano, atendiendo a la pertinencia de la letra del texto constitucional y a la futura norma en materia de educación que sea promulgada, donde sea reconocida esta modalidad de enseñanza.

\section{A Manera de Conclusiones}

Cuando se exponen y analizan algunas de las habilidades que deben formarse en los estudiantes de la carrera de Derecho, durante el curso de la carrera de forma virtual, se puede concluir:

- La incorporación de las Tics es considerada como un fenómeno social heterogéneo, la misma provoca cambios en el acceso al conocimiento, lo que repercute en el quehacer humano. El hecho de no tener acceso a las mismas dificulta la igualdad de oportunidades y genera una importante brecha cultural, económica y social, en contradicción con el derecho de acceso a la información como un derecho humano de $4^{\text {ta }}$ generación dentro de las formas de gestión en el sector empresarial.

- El problema del dominio de las habilidades y de los conocimientos asociados a éstas, supone la responsabilidad de concebir y ejecutar estratégicamente un proceso docente que en su dinámica garantice la apropiación eficiente de las mismas, al estimular y potenciar el desarrollo individual e integral del estudiante de la carrera de Derecho para interactuar con las Tics.

- Las habilidades siempre serán, resultado del aprendizaje y éstas se desarrollan en el proceso de interacción del estudiante de Derecho (sujeto) con la sociedad, a partir del silogismo que se produce, de la confrontación de la teoría y la práctica, que le permita interactuar con las Tics en los EVA.

- La articulación de un programa en la carrera de Derecho debe estar orientado a formar habilidades, que le propicien al estudiante las herramientas, los conocimientos y la cultura adecuada para el futuro ejercicio como profesional, todo ello desde el ámbito universitario para hacerlo más competente en las habilidades básicas en el mercado laboral en el siglo XXI donde se pondera el profesional competente, que domine las Tics.

- La educación en Cuba como sistema, deberá a futuro ser regulada en un cuerpo jurídico con rango de Ley, cumplimentándose el desarrollo normativo del país en la materia educativa, donde se reconozca la educación virtual en el pregrado y posgrado, para romper el paradigma del gobierno electrónico.

- A la luz de las revisiones teóricas realizadas se ha evidenciado que las transformaciones de este siglo XXI, responden a los múltiples cambios impuestos por uso de las infotecnologías en los EVA. Las relaciones que nacen en este nuevo contexto conceptualizado como "sociedad de la información", tiende a hacer posible pensar en nuevas formas de construcción del conocimiento compartido. Permiten demostrar que se está 
configurando como una nueva forma de organización, participación, movilización y colaboración.

\section{Referencias Bibliográficas}

AA.VV. (2011) Impacto de las Tecnologías de la Información y las Comunicaciones: Una mirada en actividades formativas, Revista electrónica de Veterinaria REDVET, número 7, España. Disponible en: http://www.veterinaria.org/revistas/redvet.

AA.VV. (2012) Curso virtual de redacción científica e infotecnología sobre la plataforma Moodle: resultados y experiencias. Píxel-Bit en, Revista de Medios y Educación, número 41, España. Disponible en: http://www.sav.us.es/pixelbit.

AA.VV. (2010) Curso de Infotecnología y Redacción Científica: El B-learning para profesionales de las Ciencias Agropecuarias, Revista electrónica de Veterinaria REDVET, número 11, España. Disponible en:

http://www.veterinaria.org/revistas/redvet/n111112.html.

AA. VV. (2008) La gestión didáctica en la infotecnología. Una experiencia en la Universidad de Cienfuegos, Revista Biblos, número 31, España.

AA. VV. (2010) Efectos de las tecnologías de la información y la comunicación sobre los derechos humanos, Serie Derechos Humanos Emergentes, Instituto de Derechos Humanos de Catalunya, España.

Antúnez Sánchez, A. y Rondón Valdés, J. (2016) La formación de habilidades en los estudiantes de la Carrera de Derecho. Mapas conceptuales. aprendizaje cooperativo. un recurso indispensable en la formación universitaria, Universidad de Valencia, España. Antúnez Sánchez, A. (2016) El uso de las infotecnologías para desarrollar el capital humano, Revista de Jure, Brasil.

Antúnez Sánchez, A. (2016) La empresa de alta tecnología. Una visión desde el Derecho en Cuba, Journal of humanities and social science, India.

Antúnez Sánchez, A. (2016) El modelo de la triple hélice y el desarrollo sostenible (sin publicar)

Aneca (2007) Libro Blanco del Título de Grado en Derecho, Madrid. Disponible en: http://www.aneca.es/activin/docs/libroblanco derecho def.pdf

Álvarez, I., (1999) Tesis Doctoral: El proceso y sus movimientos: Modelo de la Dinámica del Proceso Docente Educativo en la Educación superior, Universidad de Oriente, Cuba.

Albornoz, M (2001) La Política Científica y Tecnológica en América Latina Frente al Desafío del Pensamiento Único, REDES, número10, Buenos Aires.

Agirreazkuenaga, I. y Chinchilla, C. (2001) El uso de medios electrónicos informáticos y telemáticos en el ámbito de las Administraciones Públicas, Revista Española de Derecho Administrativo, número 109, Madrid.

Bates, T. (2001) Cómo gestionar el cambio tecnológico, Editorial Gedisa, Barcelona, pp.25-45.

Barrio, F. (2008) Sobre la existencia del Derecho Informático, Revista Electrónica de Derecho Informático, número 121, España. Disponible en: http://www.alfaredi.org/rdi-articulo.shtml?x=10726.

Bauzá Reilly, M. (1995) La informática en la investigación y enseñanza del Derecho, Revista de la Facultad de Derecho, número 7, Montevideo, pp.31-44. 
Berge, L. Z. Collins, M. \& Dougherty, K. (2000) Design Guidelines for Web-Based Courses, en Beverly Abbey (Ed.) Instructional and Cognitive Impacts of WebBased Education. Hershey, PA: Idea Group Publishing.

Belinchón Romo, M.R. (2011) Nuevas técnicas de aprendizaje aplicadas en el ámbito de la enseñanza del Derecho: el trabajo en grupo como parte del aprendizaje colaborativo, Universidad Complutense de Madrid, España.

Bustos, A. \& Coll, C. (2010) Los entornos virtuales como espacios de enseñanza y aprendizaje. Una perspectiva psicoeducativa para su caracterización y análisis, en Revista Mexicana de Investigación Educativa, número 44, México. pp.163-84. Disponible en: http://scielo.unam.mx/pdf/rmie/v15n44/v15n44a9.pdf,

Brunner J. (2000) Educación: Escenarios de Futuro. Nuevas Tecnologías y Sociedad de la Información. Preal-Fundación Chile (manuscrito)

Cabero Almenara, J. \& Llorente, M.C. (2005) Las plataformas virtuales en el ámbito de la teleformación. Revista Electrónica Alternativas de Educación y Comunicación, Argentina. Disponible en: http://www.e-alternativas.edu.ar/

Cabero Almenara, J. (2006) Comunidades virtuales para el aprendizaje. Su utilización en la enseñanza, EDUTEC. Revista Electrónica de Tecnología Educativa, número 20, España. Disponible en:

http://edutec.rediris.es/Revelec2/revelec20/cabero20.htm(10/08/2013).

Cabero Almenara, J. \& Llorente, M.C. (2008). La formación semipresencial a través de redes telemáticas (blended learning), Editorial Da Vinci, Mataró.

Cabero Almenara, J. (2010) Los retos de la integración de las TICs en los procesos educativos. Límites y posibilidades, Revista Perspectiva Educacional, número 1, Chile.

Cabero Almenara, J. (2014) Nuevos escenarios tecnológicos para innovar en la educación, I Seminario Iberoamericano de Innovación Docente de la Universidad Pablo de Olavide, España.

Cabero Almenara, J. (2006) Bases pedagógicas del E-learning, Revista de Universidad y Sociedad del Conocimiento, número 1, España.

Cabero Almenara, J. (2007) Las necesidades de las TIC en el ámbito educativo: oportunidades, riesgos y necesidades, Revista Tecnología y Comunicación Educativa, número 45, España.

Castells, M. (2002) La dimensión cultural de Internet. Universitat Oberta de Catalunya, España. Disponible en: http://www.uoc.edu/culturaxxi/esp/a

Cobas Cobiella, M. E. y Guillen Catalán, R. (2008) La práctica del Derecho a través de los despachos virtuales, Universidad de Valencia, España.

Cobas Cobiella, M. E. (2014) El aprendizaje cooperativo: una competencia imprescindible para la formación de los juristas, Revista Boliviana de Derecho, Bolivia, pp.604-621. Cirilo Aguilar, B. (2011) Educación virtual: fractura de un paradigma, Revista Dimensión Empresarial, número 1, México, pp.74-79.

Cebrián, M. (2008) La web 2.0 como red social de comunicación e información, Revista Estudios sobre el Mensaje Periodístico, número 14, Madrid, pp.345-361.

Cerrillo I Martínez, A. (2007) Las tecnologías de la información y el conocimiento al servicio de la justicia iberoamericana en el siglo XXI, Revista Internet, Dret i Política, número 4, España.

Díaz, B. (1992) Didáctica: Aportes para una Polémica, Editorial Aique, Argentina.

Delpiazzo, C. (2000) El Derecho telemático: respuesta a la convergencia tecnológica, en VII Congreso Iberoamericano de Derecho e Informática, Lima, p.54 y ss. 
Delpiazzo, C. (1989) Información, Informática y Derecho, Editorial A.M.F., Montevideo, p.67 y ss.

Delpiazzo, C. y Viega, M. (2009) Lecciones de Derecho Telemático, tomos I y II, Editorial FCU, Montevideo.

Delpiazzo, C. (2004) ¿Hacia dónde va el Derecho de Internet?, Derecho Informático Tomo IV, Editorial F.C.U., Montevideo, pp.247 y ss.

Delgado García, M. (2010) La enseñanza virtual del Derecho, Universidad Popular Autónoma del Estado de Puebla, México.

Duart, J. M. y Sangrà, A. (2000) Aprender en la virtualidad, Editorial Gedisa, Barcelona. Olivera, N. (2011) Estado de la cuestión en la relación entre Derecho e informática, Revista de Derecho y Nuevas Tecnologías, Argentina.

Donini, A. (2003) La gestión universitaria en el siglo XXI. Desafíos de la sociedad del conocimiento a las políticas académicas y científicas, Publicaciones Universidad de Belgrano, Buenos Aires, pp.1-75.

Fandos, M. (2003) Tesis Doctoral: Formación basada en las Tecnologías de la Información y Comunicación: Análisis didáctico del proceso de enseñanza-aprendizaje, Facultad de Ciencias de la Educación, Tarragona.

Fernández Bulté, J. (1997) Filosofía del Derecho, Editorial Félix Varela, La Habana, p.1521.

Fernández Viciedo, Y. (2014) Félix Varela Morales y el origen de la enseñanza del constitucionalismo en Cuba (1818-1824), Revista de Historia del Derecho, número 47, Buenos Aires, pp. 33-57.

Flores, O. (2012) TIC y docencia universitaria: ¿cambian las metodologías docentes según el grado de presencialidad de las asignaturas? El caso de la Universidad de Lleida. Revista Pixel-Bit de Medios y Educación, número 41, España. Disponible en: http://acdc.sav.us.es/pixelbit/images/stories/A11 010 premaq-preprint.pdf

Echeverría, J. (2000) Educación y Tecnología Telemáticas, Revista Iberoamericana de Educación, número 24, España.

Etzkowitz H. y Leydesdorff L. (1997) University and the Global Knowledge Economy. A Triple Helix of University- Industry- Government Relations. Pinter Publishers, London. López de la Madrid, M.C. (2006) Uso de las TIC en la educación superior de México. Un estudio de caso, Revista Apertura, número 7, Guadalajara.

Lanza, M. (2002) Las tecnologías de la información y comunicación como un instrumento para el desarrollo, PNUMA.

Lee, F.; Carbonell, S.; Toricella, R. (2008) Infotecnología: Ia cultura informacional para el trabajo en la Web, Editorial Universitaria, La Habana, p.49. Disponible en:

http://libros.metabiblioteca.org/bitstream/001/486/1/informacion en web.pdf Liliana Coria, S. (2017) Nuevo paradigma? Cuarta revolución industrial, Revista Iberoamericana de Derecho Ambiental y Recursos Naturales, número 23, Argentina.

Llaneza, P. (2010) Derechos fundamentales e Internet, Revista Cuadernos de comunicación e innovación, número 85, Madrid, pp.54-57.

Galgano F. (2005) La globalización en el espejo del derecho, Editorial Rubinzal-Culzoni Editores, Argentina.

García, L. Ruiz, M. Domínguez, D. (2007) De la Educación a Distancia a la Educación Virtual, Editorial Ariel, España, p.20 
González Losada, S. (2010) Análisis de las competencias de los estudiantes de Derecho en los nuevos planes de estudio de Andalucía en el EEES, Revista de Educación, número 12 , Huelva.

González De La Fe, T. (2009) El modelo de triple hélice de relaciones universidad, industria y gobierno: un análisis crítico, Revista Arbor Ciencia, Pensamiento y Cultura, número 378, p.739-755.

Madrigal Jiménez, R. (2005) El procedimiento judicial electrónico, Revista de Derecho y Tecnologías de la Información, número 3, UNED, Costa Rica.

Morales García, Ó. (2002) Delincuencia informática. Problemas de responsabilidad, Cuadernos de Derecho Judicial, número 9, Granada.

Novak, J.D. (1988) Aprendiendo a aprender, Editorial Martínez Roca, Barcelona.

Nieto Göller, R. (2012) Educación virtual o virtualidad de la educación, Revista Historia de la Educación Latinoamericana, número 19, Argentina. pp.137-150

Plan de Estudio A especialidad de Derecho. La Habana, Cuba.

Plan de Estudio B especialidad de Derecho. La Habana, Cuba.

Plan de Estudio C especialidad de Derecho. La Habana, Cuba.

Plan de Estudio C perfeccionado especialidad de Derecho. La Habana, Cuba.

Plan de Estudio D especialidad de Derecho. La Habana, Cuba. 2008.

Paredes Labrac, J. (2010) Usos educativos de la telemática, Universidad Autónoma de Madrid, España.

Pastor Sánchez, J. (1996) La gestión del conocimiento en las universidades. El nuevo documento electrónico de la tabla al hiperdocumento, Universidad de Murcia, España, pp.139-151.

Palomar Olmeda, A. (2009) El procedimiento administrativo electrónico, Curso sobre "Administración electrónica en los Gobiernos y Administraciones locales" en Huesca, España.

Pérez Luño, A. (1998) Internet y el Derecho, Revista Iberoamericana de Informática y Derecho, números 19-22, Mérida, p.722.

Pérez Luño, A. (1996) Manual de informática y Derecho, Editorial Ariel, Barcelona, 1996.

Pérez Luño, A. (2004) ¿Ciberciudadani@ o Ciudadani@.Com?, Editorial Gedisa, Barcelona.

Peña, D. (2001) El Derecho del Ciberespacio, Fundamentación Tecnológica en el Análisis del Derecho, Revista Alfa-Redi, número 37, España.

Piñar Mañas, J. L. (2007) Revolución Tecnológica, Derecho Administrativo y Administración Pública. Notas provisionales para una reflexión, Editorial Civitas, Madrid, p. 66 y 67.

Platero Alcón, A. (2015) El derecho al olvido en internet. El fenómeno de los motores de búsqueda, Revista Opinión Jurídica, número 29, Colombia, pp.243-260.

Salinas, J. (2004) Innovación docente y uso de las TIC en la enseñanza universitaria.

Revista de Universidad y Sociedad del Conocimiento, número 1, España. Disponible en: http://www.uoc.edu/rusc/dt/esp/salinas1104.pdf

Salinas Arata, A. (2012) Tesis de Maestría: Las tecnologías de la información y el Derecho, Universidad Pontificia de Perú, Lima.

Romeo Casabona, C. (2006) El Cibercrimen. Nuevos retos jurídico-penales, nuevas respuestas político-criminales, Editorial Comares, España. 
Sanz Larruga, F. (2009) Documentos y archivos electrónicos, $1^{\text {ra }}$ edición, Editorial Thonson. Civitas, España.

Soler, Yolanda; Lezcano, M. (2011) Curso de Redacción Científica e Infotecnología. Revista Cognición. No. 26, España.

Soler, Y.; Antúnez, G.; Ramírez, W.; Flores, A. (2015) La Web 2.0 en la producción de contenidos para la capacitación del profesional veterinario. RedVet, número 11, España.

Stable-Gutiérrez, C. (2006) Impacto de la infotecnología en el proceso investigativo, Revista ICIDCA, número 2, España, pp.18-26. Disponible en:

http://www.redalyc.org/pdf/2231/223120664003.pdf

Olivera, N. (2012) Estado de la cuestión en la relación entre Derecho e Informática, Revista Anales, Universidad de La Plata, Argentina.

UNESCO (2009) Conferencia Mundial de Educación Superior 2009: Las Nuevas Dinámicas de la Educación Superior y de la Investigación para el Cambio Social y el Desarrollo, Paris.

Teare, R. y Davies, Sandelands, E. (2002) Organizaciones que aprenden formación virtual, Editorial Gedisa, Barcelona.

Talizina, N. (1992) La formación de la actividad cognoscitiva de los escolares, Editorial Ángeles editores, México.

Tesoro, J. (2008) La Carta Iberoamericana de Gobierno Electrónico como hito hacia un nuevo paradigma de e-gobierno. España. Disponible en:

http://www.gobiernoelectronico.org/node/5923

Torres Cuevas, E. (2002) Félix Varela: los orígenes de la ciencia y conciencia cubana, Editorial Ciencias Sociales, La Habana, p. 157.

Informe mundial de las Naciones Unidas sobre el sector público (2003) La Encrucijada del Gobierno Electrónico. Disponible en:

http://www.cinu.org.mx/prensa/comunicados/2003/PR03108 Inf e gov

Rico Carrillo, M. (2007) Derecho de las nuevas tecnologías, Editorial La Rocca, Buenos Aires.

Reyes Krafft, A. (2002) Tesis Doctoral: La Firma Electrónica y las entidades Certificadoras, Universidad Panamericana, México.

Sánchez Rodríguez, F. (2005) Las nuevas tecnologías de la información y la comunicación en la Administración Pública como paradigmas de un servicio público eficaz y eficiente prestado a los ciudadanos, en Punzón Moraleda, J. Administraciones Públicas y nuevas tecnologías, Valladolid.

Quesada, K. y Rooney, F. (2016) Las incubadoras de abogados para mejorar la enseñanza del Derecho y el acceso a la justicia, Revista Derecho y Cambio Social, Perú. Kelsen, H. (2009) Teoría pura del Derecho, Editorial Porrúa, 16a edición, México.

Kuhn, T. (1975) Segundos pensamientos sobre paradigmas, Madrid.

Kuhn, T. (1995) La estructura de las revoluciones científicas, Fondo de Cultura Económica, México.

Witker Velázquez, J. (1995) Antología de estudios sobre la enseñanza del Derecho, UNAM, México. Disponible en: hptt//www.juridicas.unam.mx/inst/direc/datper.htm$\mathrm{p}=$ witker.htm

Vázquez, B. (2015) El uso de aplicaciones para celulares como experiencia de Mobile learning en Ciencias Agrarias, IV Taller Internacional de Educación a Distancia y Pedagogía, Bayamo. 
Varela Morales, F. (1996) Cartas a Elpidio, Tomo I, Editorial cubana, Miami, 1996, pp.34-182.

Valero Torrijos, J. (2004) Régimen Jurídico de la e-Administración. El uso de medios informáticos y telemáticos en el procedimiento administrativo, Editorial Comares, Granada.

Villar Palasí, J. (2000) Implicaciones jurídicas de Internet, Revista Saberes de Estudios Jurídicos, Económicos y Sociales, número 1, España. 